EUROPEAN UNIVERSITY INSTITUTE, FLORENCE

DEPARTMENT OF LAW

Temporal Reasoning and MAS

Clara Smith, Antonino Rotolo and Giovanni SARTor

EUI Working Paper LAW 2010/16 
This text may be downloaded for personal research purposes only. Any additional reproduction for other purposes, whether in hard copy or electronically, requires the consent of the author(s), editor(s). If cited or quoted, reference should be made to the full name of the author(s), editor(s), the title, the working paper or other series, the year, and the publisher.

ISSN 1725-6739

(C) 2010 Clara Smith, Antonino Rotolo and Giovanni Sartor

Printed in Italy

European University Institute

Badia Fiesolana

I - 50014 San Domenico di Fiesole (FI)

Italy

www.eui.eu

cadmus.eui.eu 


\section{Author contact details}

Clara Smith

Faculty of Informatics and Faculty of Law

University of La Plata

Argentina

Partially supported by PICT 2006-00842 ANPCyT

Email: csmith@info.unlp.edu.ar

Antonino Rotolo

CIRSFID

University of Bologna

Italy

Email: antonino.rotolo@unibo.it

Giovanni Sartor

European University Institute,

Florence, Italy

CIRSFID

University of Bologna

Italy

Email: giovanni.sartor@eui.eu 



\begin{abstract}
In this paper we investigate if it is possible and useful to reason about time within social/normative multi-agent systems (MAS) by taking into account the general guidelines of tense logic. We focus on the combination of special-purpose logics: we provide a formal account in which a minimal temporalization helps in reasoning about time in an abstract way. We also explore a new variant of deontic tense logic by using a hybrid tense logic. The accounts provided allow to model temporal provisions within both particular norms and general legal principles, and also help in the detection of breaches of good faith and confidence.
\end{abstract}

\title{
Keywords
}

hybrid modal logic, deontic logic, temporal reasoning, deadlines, multi agent systems 



\section{Introduction}

Both law and technology deal with temporal specifications. Relevant to the law are, among others, the prescription of actions to be accomplished within certain time, obligations with deadlines, the duration of the legal year, the duration of a leasehold in land, or an insurance policy during a specified period. For computing systems in general, finiteness and infiniteness of processes are of main interest, as well as the duration and successful termination of different kinds of atomic or complex transactions, the activity or inactivity of sessions, logins, the possibility of distributed or parallel processing, etc.

The relevance of the relation between time and deontic logic was well-settled in the seventies and eighties. Nowadays, several social and normative MAS capture diverse aspects of time mainly from a modelling point of view. However, consistent working applications of modal tense logics to those systems are still overlooked. Also, technical issues such as decidability of temporalized MAS are usually difficult to face.

For instance, the framework in [1] helps to model social constraints, roles, and states using concepts from legal and social systems, using the Event Calculus [2], a first-order formalism for reasoning about events. Time is explicitly codified inside constraints as in e.g. HoldsAt(permitted(agent, action), $t$ ) which generically specifies a permission: at time $t$ it is true that the action is permitted for the agent. Given a temporal ordering of events we can find out the set of powers, permissions, obligations and sanctions each agent has at each point in time. As a second example, consider the account in [3]. It comprises a defeasible theory based on [4] which allows expressing some aspects of FIPA semantics for Agent Communication Languages (ACL). Instants are modeled as timestamps labelling literals (a literal is an atomic proposition or its negation), e.g. 1:t is meant to stand for 'literal 1 holds at time t'.

Within both accounts, thinking back and forth along the time line may be a demanding enterprise. For example, moving along the flow of time means searching the knowledge base for rules and literals tagged with a given instant $t$, and performing the plausible deductions; then repeat with instant $t+1$ (or resp. t-1) and so on (in line, see explanations to Example 3.3 in [3]). Typical temporal formulas such as: 'it has always been the case that p' or 'it is always going to be the case that p' need back and forward searches throughout the knowledge base (see the reading of rule r10 in Example 3.3 in [3]).

Other MAS such as [5,6] handle in different ways some forms of temporal reasoning. The account in [5] for example, uses for its definition of trust some well-known concepts from dynamic logic (which can be seen as including temporal aspects.) In the survey in [7], its authors refer to various works incorporating aspects of time to overcome some inherent difficulties with standard deontic logic. For instance, the dyadic temporal operator proposed by J. A. van Eck [8] relativizes deontic statements to points in time where they should hold. The authors also refer to [9], which reduces deontic specifications to first-order temporal ones by interpreting the obligation to do $p$ as the property that $p$ must occur sometime in the future.

Theoretically speaking, the general idea of reasoning about time should extend any MAS consistently. From a computational standpoint, we believe that a minimal functionality for the automatic manipulation of time -i.e. from a basic modal perspective- should involve few technical adjustments of the MAS's underlying logic. As an added challenge, we also argue that a temporalization straightforwardly helps in the detection of breaches of good faith and confidence in MAS.

We organize the work as follows. Section 2 describes two combinations of tense logics with multimodal multi-agent logics. Section 2.1 uses a basic tense logic (understood in the classical way introduced by Prior [10]) to temporalize - in the sense given in [11, Section 2] — multi-modal MAS. This allows us to express abstract time provisions and lawful principles straightforwardly. In Section 2.2 we propose a new variant of deontic tense logic by considering an extension to modal logic called 
hybrid logic which allows us to name points in time. This way we gain a "HoldsAt" functionality. Section 2.3 extends both accounts in 2.1 and 2.2 by adding agent's goals and beliefs. In all subsections we work with examples referring to Argentinean and to Italian Law, and also with a more general example from the literature. Examples are presented throughout the paper in an increasing level of complexity. In Section 3 we mention some decidability issues regarding the logics used throughout. Some conclusions end the paper.

\section{Temporal Specifications and Norms}

Many MAS are designed as multi-modal systems $[5,12,13,14]$. Different modal operators define different agents' features such as intentions, beliefs, agency, etc. Embeddings of tense logic into the basic modal language were first studied by S. K. Thomason in the mid '70s [15]; the approach has gained popularity recently, see $[11,16,17,18]$. It is pointed out in [7] that Thomason has argued that deontic logic requires a foundation in temporal logic, reducing the obligation of $\mathrm{p}$ to a temporal statement that $\mathrm{p}$ holds in all future worlds that would be reachable.

The description of a plain, basic logic of time, restricted to a traditional Kripke-style modal perspective, and which comprises a great variety of systems [17] is usually as follows: the basic temporal language is built using two unary operators, $\mathrm{F}$ and $\mathrm{P}$, a set of propositional letters $\mathrm{p}, \mathrm{q}, \mathrm{r} . .$. , and Boolean connectives [18]. The intended interpretation of a formula Fp is 'p will be true at some Future time', and $\mathrm{Pp}$ is meant to stand for ' $\mathrm{p}$ was true at some Past time'. Duals for $\mathrm{F}$ and $\mathrm{P}$ are, respectively, G and H ('it is always Going to be the case' and 'it Has always been the case'.) This is the core language underlying the branch of modal logic called tense or temporal logic. The usual mathematical structures where temporal formulas are interpreted are bidirectional frames [18, pp. 21]. For now, let us assume a frame is a structure $\mathrm{T}=(\mathrm{T},<)$ where $\mathrm{T}$ is a set of instants of time and $<$ is a precedence relation such that if $\mathrm{s}<\mathrm{t}(\mathrm{s}, \mathrm{t} \in \mathrm{T})$ then we say that $\mathrm{s}$ is earlier than $\mathrm{t}$ [17]. The minimal pointwise tense logic is $\mathrm{K} 4$, which is complete w.r.t. the class of transitive frames. As one of our motivations is decidability, i.e. to provide decidable temporalized MAS, we will explore two possible methods for embedding temporal reasoning in multi-modal settings:

In a first possibility, we add temporal modalities to a multi-modal language for agents already including operators for deontic provisions, agency, and, possibly, agents' goals and beliefs. Since this extension does not allow for explicitly referring to time instants in the language, it looks useful for representing abstract temporal properties or constraints, or for capturing uncertain time provisions such as regulations or obligations conditioned to undetermined events.

A second possibility is to combine a multi-modal logic as mentioned above with a hybrid temporal logic (see, e.g. [18, pp. 434].) This logic explicitly identifies points in time and accounts for the moments at which an event happens, or the temporal scope of lawful provisions begins (or ends.)

\subsection{Temporal Social/Normative MAS}

The literature provides a number of different techniques for combining logics, such as products, fibring, fusion, and temporalization. Indeed, the reasoning patterns we present simply require the addition of a temporal logic on top of the logics underlying the MAS.

On account of [12]'s advice, let us work with a multi-modal approach for dealing with agents' attitudes. Assume we deal with a finite set of agents $\mathrm{A}=\{\mathrm{x}, \mathrm{y}, \mathrm{z} \ldots\}$ and a countable set $\mathrm{P}$ of atomic propositional sentences denoted by $\mathrm{p}, \mathrm{q}, \mathrm{r}$.... Complex expressions are formed syntactically from these, plus the following unary modalities we describe next.

A deontic operator $\mathrm{O}$ represents generic (legal/lawful) obligations, meaning "it is obligatory that" $[7,12]$. An operator for agency Doesx A represents successful agency i.e. agent $\mathrm{x}$ indeed brings about 
A [19]. For simplicity, we assume that in expressions like Doesx A, A denotes behavioural actions concerning only single conducts of agents such as withdrawal, inform, purchase, payment, etc. (no modalized formulas occur in the scope of Does.) $\mathrm{O}$ is taken to be a classical KD operator. The logic of Does is non-normal, closed under logical equivalence and amounts to the following schemata [20]: DoesxA $\rightarrow$ A, (DoesxA $\wedge$ DoesxB $) \rightarrow \operatorname{Doesx}(A \wedge B)$, and $\neg$ DoesxT.

We now bring in the temporal modalities for writing temporal formulas; for example, the modality $\mathrm{F}$ mentioned above will allow us to characterise future obligations, as well as the future extinction of an obligation. Before we go to the semantic details, we present two examples.

Example 1. Obligations in the future. According to art. 566 of the Argentinean Civil Code, and according to art. 1183 of the Italian CC, a deadline may be established for complying with an obligation. Similarly, the emergence of obligations (and of the corresponding rights) can be postponed to a future moment in time; or a future moment may be established for extinction of obligations. Intuitively, time works in such scenarios as a modality of the obligation; the obligation is what is being modalized.

The temporal operator $\mathrm{F}$ allows us to express that an obligation will hold sometimes in the future: $\mathrm{F}(\mathrm{O}$ Doesx A) means that "it will be true in some future time that there is the obligation for agent $\mathrm{x}$ to do A". It also allows us to express that there will be a future time in which an obligation will not hold: $\mathrm{F}(\neg \mathrm{O}($ Doesx $\mathrm{A}))$ means that "it will be true in some future time that there is no obligation for agent $\mathrm{x}$ to do A". It may also apply to conditional obligations: $\mathrm{F}(\mathrm{p} \rightarrow \mathrm{O}($ Doesx $\mathrm{A}))$ means that there will be an instant in the future when if $p$ holds then there is the obligation for agent $x$ to do A. Similarly, $F(p$ $\rightarrow \neg \mathrm{O}($ Doesx A)) means that there will be an instant in the future when if $p$ holds then there is no obligation for agent $\mathrm{x}$ to do A. As examples of this kind of normative statements consider the following:

$\mathrm{F}(\mathrm{O}$ (Doesj Pay $))$, there is a future moment in time in which agent $\mathrm{j}$ will have the obligation to pay;

$\mathrm{F}(\neg \mathrm{O}$ (Doesj Pay)), there is a future moment in time in which there is no obligation for $\mathrm{j}$ to pay;

$\mathrm{F}$ (Doesk Ask $\rightarrow$ (O Doesj Pay)), in the future there will be a moment in which, if $\mathrm{k}$ asks fo the payment then $\mathrm{j}$ has the obligation to pay;

$\mathrm{F}($ Doesj Pays $\wedge(\neg \mathrm{O}($ Doesj Pay $)))$, in the future there will be a moment in which it will be that case that $\mathrm{j}$ pays but he has no obligation to pay.

While such statements express interesting normative positions for the involved agents, they fail to capture temporally limited obligations, since they do not provide sufficient information to $\mathrm{j}$ and $\mathrm{k}$. To be able to comply with an obligation starting in the future (1), $\mathrm{j}$ needs to know what is the precise time when the obligation will start to hold (it may not be enough to know that it will hold sometime in the future.) Similarly, for being able to reject future compliance with an obligation terminating in the future (as in 2), and in, e.g. 'I will not work for you tomorrow since my work contact expires today') j needs to know when the obligation will terminate. For the obligation in (3) to be satisfied, it is sufficient that there is one future instant in which $\mathrm{k}$ ask for payment, or the payment is provided. On the contrary, usually, a conditional obligation to pay usually requires that payment is provided when one is asked for the first time (the creditor would not accept the reply: 'ok, in the future there will be an occasion when I will pay when asked, but this is not that time; try again, you may be luckier!').

These representations fail to capture the fact that an obligation usually does not hold for an instant, but it spans over a time interval, and similarly, it does not hold forever. A way to capture the persistence of an obligation (or its absence) consists in using G, the dual of F (Section 2.) The expression GA stands for "A will always hold in the future", e.g. Diesx $\rightarrow \mathrm{G}(\neg \mathrm{O}$ (Doesx A)) means that if $\mathrm{x}$ dies, s/he will never have the obligation to do A. For example, the death of a person who has been instituted as head of a life-rent extinguishes forever the creditor's obligation to pay (art. 2070 ACC, art. 1873 ICC.) Simple formulas like the former consequent $\mathrm{G}(\neg \mathrm{O}(\operatorname{Doesx} \mathrm{A}))$ are indeed powerful. This expression 
can be considered as a possible formulation for the legal institution called prescription, which in one of it forms establishes that "by the time designed by law, the debtor is free from its obligation" meaning that there will be a future moment in time in which the creditor cannot pursue her/his legal right in court (art. 4017 ACC, art. 2934 ICC.) However, to model a prescription more is required: we need to be able to represent the time point where the prescription operates, so that the obligation terminates. As we shall see in the following, for addressing the limitations just illustrated we supplement our logic with an explicit way of naming time-points and intervals.

\subsubsection{Semantics for Temporalized MAS}

Regarding the semantics of the combination of logics we propose, we follow the temporalization technique $[11,21]$ which, intuitively, amounts to place the temporal machinery on top of the MAS.

We consider both general and relativised obligations (Oa is a relativised deontic operator meaning "it is obligatory in the interest of agent a that" [22]). We restrict ourselves to well-formed atomic temporal formulas whose outermost symbol is a temporal operator. Formally, the behaviour of such a system is captured by a model $(\mathrm{T},<, \mathrm{g}, \mathrm{t} 0)$. The outer frame $(\mathrm{T},<)$ corresponds to the temporal evolution of the system; $t 0 \in \mathrm{T}$ is the initial point in time. The system evolves through time in the sense that new generic/individual obligations/permissions are settled while some others become obsolete or prescribe. Following, $g$ is a function such that for every $t \in T, g(t)$ returns a multirelational model for the MAS itself. According to $[14,20]$ we build a multi-relational frame of the form:

$\mathrm{F}=<\mathrm{A}, \mathrm{W}, \mathrm{O},\{\mathrm{Oi}\} \mathrm{i} \in \mathrm{G},\{\mathrm{Di}\} \mathrm{i} \in \mathrm{G}>$

where:

A is a finite set of agents;

$\mathrm{W}$ is a set of situations, or possible worlds;

$\mathrm{O}$ is the accessibility relation for the deontic operator, which is serial (standard KD semantics);

$\{\mathrm{O} i\} \mathrm{i} \in \mathrm{G}$ is the set of accessibility relations w.r.t. relativised obligations, which are serial (usual KDn semantics);

$\{\mathrm{Di}\} \mathrm{i} \in \mathrm{G}$ is a family of sets of accessibility relations Di w.r.t. Does; which are reflexive, serial, and pointwise closed under intersection. 
A multi-relational model is, in its turn, a structure of the form:

$\mathrm{M}=<\mathrm{F}, \mathrm{V}>$

where:

$\mathrm{F}$ is a multi-relational frame as above, and

$\mathrm{V}$ is a valuation function defined as follows:

standard Boolean conditions;

$\mathrm{V}(\mathrm{w}, \mathrm{O} A)=1$ iff $\forall \mathrm{v}$ (if $\mathrm{wOv}$ then $\mathrm{V}(\mathrm{v}, \mathrm{A})=1$ );

$\mathrm{V}(\mathrm{w}, \mathrm{Oi} \mathrm{A})=1$ iff $\forall \mathrm{v}$ (if wOiv then $\mathrm{V}(\mathrm{v}, \mathrm{A})=1$ );

$\mathrm{V}(\mathrm{w}$, Doesi $\mathrm{A})=1$ iff $\exists \operatorname{Di} \in \operatorname{Di}$ such that $\forall \mathrm{v}(\mathrm{wDiv}$ iff $\mathrm{V}(\mathrm{v}, \mathrm{A})=1)$;

This way, according to the notation in [12], $(\mathrm{T},<, \mathrm{g}, \mathrm{t} 0)$ amounts to a model for the logic of a temporalized MAS. Notice that although in the resulting logic we can write temporal formulas, the formulas of the nested MAS (which have no temporal operators) are unambiguously evaluated w.r.t. a given model $\mathrm{g}(\mathrm{t})$.

\subsection{Temporal Reasoning and the 'Holds At' Functionality}

Terms can be certain or uncertain. This classification takes into account the moment at which the key event happens. If such moment is known by the time of arrangement (for example, at contract formation) the term is said to be certain, otherwise it is uncertain: although it will necessary happen, we do not know when it will (for example, a death.)

So far, we have been working with points in time throughout Section 2.1. However, we are not able to clearly identify such points in the account. This feature may appear to be a drawback for applications which consider selected time points; it is sometimes important to reason about what is going on at particular moments. Effective for representing provisions such as those in Section 2.1, basic temporal logic is plain and straightforward; it does not provide an analogous tool as the HoldsAt(A,i) predicate in [1] or the 1:t tagged literal in [3]. As Blackburn and Franceschet point out [11,18], hybrid logics -an extension of modal logics- helps us to deal with particular moments.

We will be working next with a hybrid temporal logic, which is an extension of the basic temporal logic used in Section 2.1. This logic will subsequently be the one used to temporalize. A hybrid temporal language helps us to treat points in time as "first class objects", by naming them individually and directly. For example, if we are working with the domain of months, we can assign the names $\mathrm{j}, \mathrm{f}$, $\mathrm{m}, \mathrm{a}, \ldots$ to particular worlds in the domain of months the same way we give constant names to refer to specific elements in any domain (as we do with ' 0 ' and ' 1 ' within the integers.) Being able to locate points in time leads us to ask wether a certain event will happen in a given future moment, like in: "will we be having a rainy month of April?", or if it has happened in a past time, as in: "were the company flights rescheduled in August?". Finally, we may want to give a name to the current moment because, e.g., it is the moment in which we are in a position to fulfil our and other parties' expectations; assuming a domain of days we can propose: "let's sign the contract today, December 27th", or: "you may stay and start working from now, December 27th, on". This possibility looks necessary in order to be able to indicate a date in lawful acts.

For dealing with particular instants of time in our account, we proceed, technically, as follows. We take the basic modal language in Section 2.1 and add a second sort of atomic formulas. These atoms are called nominals, written $\mathrm{i}, \mathrm{j}, \mathrm{k}$... . A nominal names a world, or point, by being true in that point 
and nowhere else; so if $i$ is a nominal the formula $i$ holds if and only if the current location is named $i$. Old and new atoms are combined to form complex formulas in the usual way (as in $i \wedge p$.) For direct access to worlds, basic hybrid logics provides a '@' operator. This operator, called a satisfaction operator, allows us to build formulas such as @iA, which is true at any point in a model if and only if A is satisfied at the unique point named by i. Thus we may eventually have a family of @i operators in our language; hybrid logics are essentially multi-modal logics. Satisfaction operators are normal modal operators. Note that @iA expressions play the same role of $\operatorname{HoldsAt}(\mathrm{A}, \mathrm{i})$ propositions and 1:t facts. Hybrid logics may also include the downarrow binder $\downarrow_{i}$. which creates a name $i$ and assigns it to the currently evaluated world. Two examples follow.

Example 2. Hybrid-temporalised MAS; termed obligations, and claimable obligations. Suppose we

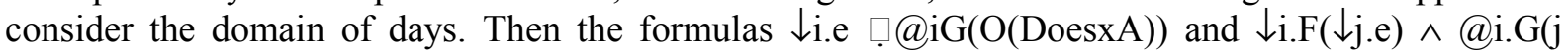
$\square \mathrm{G} \neg(\square$ Doesx A )) stand for "when the event e occurs, from that day on it will be the case that agent $\mathrm{x}$ is obliged to do A", and "from now on, there will be a day in which the event e will happen, and from that day on it will be the case that there is no obligation for $\mathrm{x}$ to do A", respectively. The fact that an obligation A is claimable by time i can be written as @ $\mathrm{i} \square \mathrm{A}$.

Example 3. Hybrid-temporalised MAS with relativised obligations; payment made before due time (Art 571 ACC.) The sentence

$$
\begin{aligned}
& (\downarrow \text { i. }(\text { DoesxPay }) \wedge @ \mathrm{iF}(\downarrow j \mathrm{j} . \mathrm{Oy}(\text { DoesxPay }))) \square \\
& @ \mathrm{i} \neg \mathrm{G}(\square \mathrm{x} \text { Doesy Reimburse })^{1}
\end{aligned}
$$

establishes that if agent $\mathrm{x}$ pays on day $\mathrm{i}$ and there is a future day in which it is obligatory in the interest of agent $y$ that $x$ pays (Oy expresses a relativised obligation, namely, the obligation meant to satisfy the interest of agent $y$, see [22]), then there is no obligation for $y$ to reimburse such payment. It is plain to see that by using a hybrid logic we keep the modal temporal reasoning facilities and add a "HoldsAt" functionality.

As another example, consider the scenario where agent $\mathrm{x}$ works for agent $\mathrm{y}$, starting on day i. This can be represented as @iG(O(Doesx Workfor(y))). Similarly, the fact that x will not be obliged to work for $y$ from day $j$ on can be written as $@ j \neg G(O($ Doesx Workfor $(y)))$. Note that though both prescriptions make sense separately, they lead to a contradictory situation: it cannot be the case that in the future there are instants in wich $\mathrm{x}$ is obliged to work and obliged not to work for $\mathrm{y}$. To avoid such type of contradictions, and to be able to expand our framework with obligations spanning until an event terminates them, we have to provide a futher extension to our model. We will make use of the until modal operator.

Further extension. So far, we have been working with abstract temporal properties, and with uncertain time provisions. Then we added the possibility of explicitly referring to points in time. But for several MAS applications all these appear to be not enough. For example, we may want to be able to formally write sentences like "if agent a damages agent $b$, until the obligation is extinguished it is obligatory that a pays b", which responds to the more general temporal pattern " $p$ will be the case, and until that happens, q will hold": $\mathrm{U}(\mathrm{p}, \mathrm{q})$. Those p properties are called guarantee properties in the computational literature [18]. While U searches forwards along a linear pointwise flow of time, the binary operator since: $\mathrm{S}(\mathrm{p}, \mathrm{q})$ is used to look backwards.

\footnotetext{
We may have also formalized this consequent as "there is no obligation in the interest of agent $\mathrm{x}$ for $\mathrm{y}$ to reimburse not in time $i$, neither after $i$ and before $j$ ", meaning that the payment must have been done by time $\mathrm{j}$.
} 
Let us see how to fit these operators in our hybrid-temporal multi-modal account. The satisfaction definition for $\mathrm{U}$ is:

$\mathrm{t} \Vdash \mathrm{U}(\phi, \psi)$ iff there is a $\mathrm{v}>\mathrm{t}$ such that $\mathrm{v} \Vdash \phi$ and for all s with $\mathrm{t}<\mathrm{s}<\mathrm{v}$ : $\mathrm{s} \Vdash \Psi$

which it is to be interpreted on frames with structure $(\mathrm{T},<)$ as stated in the Introduction. Therefore, the above semantic definition for $\mathrm{U}$ clearly suits the temporal intended behaviour of the model $(\mathrm{T},<, \mathrm{g}, \mathrm{t} 0)$ we gave in Section 2.1.1. (Correspondingly, define $\mathrm{S}$ as a search in the opposite direction in the time line.)

It is well known that $\mathrm{U}$ and $\mathrm{S}$ are not definable in the basic modal language (see e.g. [17,18]). Moreover, proposition 7.10 in [18] proves that $U$ is not definable over the real numbers with $<$, using $\mathrm{F}$ and $\mathrm{P}$. On the other hand, $\mathrm{F}$ and $\mathrm{P}$ are definable in a language with $\mathrm{U}$ and $\mathrm{S}$, thus such a language is stronger than the basic temporal language. Therefore, let us extend our language with the binary operators U and S. U and S-formulas are built straightforwardly from Boolean connectives and propositions in the usual way.

Example 4. Hybrid-temporal MAS with Until; Deal example. Agents a and b make the following deal: "a has the obligation to pay b, until a does pay or June arrives". Formally, this deal can be expressed as $\mathrm{U}((($ DoesaPay $) \vee \mathrm{June}), \mathrm{Ob}($ Doesa Pay)); recall that June is here a nominal (and nominals play the role of propositional constants.) A proper payment before the forthcoming month of June will release agent a from his obligation. It follows from the example (and from U's satisfaction definition) that the obligation to pay holds in all months previous to June and up to the month a pays (if on time); when June comes, the obligation will no longer hold.

\subsection{Temporal Reasoning: MAS with Beliefs and Goals}

We next test with a couple of examples our intuitions regarding the usefulness of the reasoning about time within MAS for detecting breaches of good faith and confidence. Objective good faith is usually described as correctness, i.e., as a standard of behaviour which is to be recognised not only with reference to legal rules, but also with reference to social norms or background rules (namely, to norms and rules that are not explicitly stated within a legal code) $[23,24,25,26,27]$. Article 1175 of the Italian Civil Code states that one should follow the rules of correctness. These require that the trustee takes care to avoid damaging the trustor, i.e., the trustee should take into account the legitimate interest and expectations of the other party.

For working with the next example we extend the language with an operator for goals: Goalx A. It aims to stand for "agent $\mathrm{x}$ has goal $\mathrm{A}$ "2, where $\mathrm{A}$ is a proposition.

Example 5. Temporalised MAS with Goal operators (in [28], revisited.) Agent a is debtor of agent b. At the time of prescription of b's action against $a, b$ asks a to pay his debt, making a know he will be sued otherwise. Agent a says that he has no money at the time but that he will have enough in six months, which largely exceeds the prescription limit. $b$ accepts to informally relax the deal. Six months later, a refuses to pay $b$. Then $b$ sues $a$.

2 As classically established, Goali is a Kn operator. The multi-relational frame already defined should correspondingly be extended with $\{\mathrm{Gi}\} \mathrm{i} \in \mathrm{G}$, a set of accessibility relations w.r.t. goals. 
Discussion. At time of prescription the following facts hold:

$\mathrm{Ob}$ (Doesa Pay), it is obligatory in the interest of agent $\mathrm{b}$ that a pays his debt;

Goala( $\neg$ Pay), agent a has as (inner) goal not to pay;

$\mathrm{Ob}$ (Doesa Pay) $\square \mathrm{Ob}$ (Goala(Doesa Pay)), when there is an obligation, then the obliged agent has the good-faith obligation to have the goal to accomplish (correctness rule);

$\mathrm{G}(\neg \mathrm{Ob}$ (Doesa Pay)), from now on it will always be the case that there is no obligation of a to pay, as the obligation prescribes;

G(Goala( $\neg$ Pay)), it will always be the case that a has as goal not to pay.

From the two first facts we get that a's goal of not paying contradicts his obligation to pay. This is an indicative of a breach of a's good faith by the time of prescription of b's action. Six months later it holds that $\neg$ (DoesaPay), which conforms the last fact in the list above and also contradicts the deal.

Following, let us consider what happens to the correctness rule Ob(Doesa Pay) $\rightarrow \mathrm{Ob}$ (Goala(Doesa Pay)): by not having the goal of paying at the time he had to pay, a has violated the correctness obligation. However, when the main obligation expires also the correctness obligation does not apply any longer. Although there are indicatives of a's not acting in good faith, b would lose the case. A lawyer would indeed say that, from the moment the action prescribes, the obligation becomes a natural obligation (see e.g. Art. 515 ACC.) -this means that its fulfilment is founded now not in law, but in a principle of equity (this means that a is not obliged to pay, but if he does then he has no right to have his money back.)

Some important aspects of good faith and trust may be analysed on the basis of the concept of subjective good faith, which requires the trustor's sincere belief that he is doing things without damaging others' rights. For discussing this richer scenario, extend again our language with the doxastic modality Belx A, which is meant to represent that $\mathrm{x}$ has the belief that $\mathrm{A} 3$. Let us now see an example illustrating how to combine hybrid temporal logic with agent's goals and beliefs.

Example 6. Hybrid-temporalised MAS with Until, and Goal operators (in [28, once again, revisited].) Agent $a$ is debtor of agent $b$. By $t 1$, the time of prescription of b's action against $a, b$ asks a to pay his debt, making a know he will be sued otherwise. Agent a says that he has no money now but that he will have enough in six months, by $\mathrm{t} 7$. Agent $\mathrm{b}$ accepts to relax the deal. By $\mathrm{t}$, a refuses to pay $\mathrm{b}$. Then $\mathrm{b}$ sues $\mathrm{a}$.

Discussion. The following hold:

$\mathrm{U}($ Expires(Ob(Doesa Pay), $\mathrm{Ob}($ Doesa Pay)), it is obligatory in the interest of agent $\mathrm{b}$ that a pays his debt, until this obligation expires;

Expires(Ob(Doesa Pay)) $\square \mathrm{G}(\neg \mathrm{Ob}$ (Doesa Pay)), when the obligation expires it does not hold any longer;

$\mathrm{Ob}$ (Doesa Pay) $\square \mathrm{Ob}($ Goala(Doesa Pay)), the aforementioned correctness rule;

@t1 Goala( $\neg$ Pay), agent a has as (inner) goal not to pay;

@ $\mathrm{t} 1 \mathrm{G}(\mathrm{Goala}(\neg$ Pay $))$, it will always be the case that a has as goal not to pay;

@t7 Expires(Ob(Doesa Pay)); the deal expires at t7.

3 Respectively, extend again the multi-relational frame, now with a set of accessibility relations w.r.t. Bel, which are transitive, Euclidean and serial. 
By time $\mathrm{t} 7$ agent a has achieved his goals: he will no longer be obliged to pay ( $\mathrm{G}(\neg \mathrm{Ob}$ (Doesa Pay)), and he will never have to pay, because the obligation has expired (i.e. the deal is over.) Let us assume on the contrary that $\mathrm{b}$ sues $\mathrm{a}$, at a time $\mathrm{t} 0$ before the expiration time $\mathrm{t} 1$. In this case, it holds that:

$$
\begin{aligned}
& \text { @ t0Ob(Doesa Pay); } \\
& \text { @t0-7DoesaPay; } \\
& \text { t0Goala( } \neg \text { DoesaPay); } \\
& \text { t0Ob(Goala(Doesa Pay)). }
\end{aligned}
$$

Consequently, at t0, agent a violates both the obligation to pay and the good-faith correctness rule to have the goal to pay. Note here that the corresponding sanction can be even more severe that it would be if he failed to accomplish his obligation in good faith, i.e., without having the goal to do so.

\section{Decidability Issues}

Regarding issues of decidability w.r.t. to our proposals in this work, it is well known that the logic that results from the combination through temporalization of a decidable logic and a basic tense logic is also decidable. PSPACE algorithms have been devised for a number of well-known logics including the temporal counterparts of $\mathrm{K}, \mathrm{T}, \mathrm{K} 4$ and S4. Theorem 7.1 in [18, pp. 436] settles that the satisfiability problem for basic hybrid logics is PSPACE-complete. Finite model checking algorithms for temporalizations -in the significance given in this paper- are available in [11]. Moreover, that paper suggests a temporalization using a hybrid logic. Furthermore, [29] provides terminating tableau systems for a number on non-transitive hybrid logics extending $\mathrm{K}$ such as the logic of irreflexive, antisymmetric frames; also [29] provides (for hybrid tense logic enriched with a universal modality) a terminating tableau calculus for the logic of transitive frames. Finally, $\mathrm{U}$ and S-formulas are complete w.r.t. frames $(\mathrm{T},<)$ such that $(\mathrm{T},<)$ is a well-ordered flow of time called Dedekind complete order, such as the total order of the natural numbers [11]. We consider all this background provides a strong preliminary platform where to build proof procedures for temporalized MAS such as those in this paper.

\section{Concluding Remarks}

In this paper we addressed some forms of temporal reasoning within MAS, and suggested a perspective on how these combinations can be used for modelling aspects of time within lawful provisions, obligations, legal principles, and good faith. The main collaboration is the new variant of deontic tense logic using hybrid logics. The temporalizations we suggested provide evidence that minimal adjustments are required for existing frameworks such as those in, e.g., [14] to deal with both a minimal temporal reasoning functionality and a 'HoldsAt' device. We applied a methodology which falls under the general name of combination of logics (see [11,30]): we combined special-purpose logics of restricted expressive power.

The systems we obtain are simple from the logical point of view; their simplicity is support for their usefulness and robustness, and also keeps the systems manageable, decidable, and suitable for further studies and extensions. The use of $U$ leads us to consider an even more prominent role for time periods within normative MAS; we have treated intervals not as primitive objects but built them on top of a more basic element, the point in time. We did not include complex definitions for concepts such as trust, agreement, commitment, roles, or speech acts, which directly increase the complexity of the MAS. These further extensions, i.e. how to put to work all (or some of) these concepts together, are matter of our future investigations. 


\section{References}

[1] A. Artikis, J. Pitt, M. Sergot. Animated Specifications of Computational Societies. AAMAS 2002, ACM.

[2] R. Kowalski, M. Sergot. A logic-based calculus of events. New Generation computing, 4(1):67-96, 1986.

[3] G. Boella, G. Governatori, J. Hulstijn, R. Riveret, A. Rotolo, L. van der Torre. Time and Defeasibility in FIPA ACL Semantics. WLIAMAS 08.

[4] G. Governatori, G. Sartor, A. Rotolo. Temporalised normative positions in defeasible logic. Proceedings of ICAIL 2005, ACM Press.

[5] C. Castelfranchi, R. Falcone, 'Social trust: a cognitive approach', in Trust and Deception in Virtual Societies, Springer, 2001.

[6] R. Demolombe, C-J. Liau. A Logic of Graded Trust and Belief Fusion. 4th Wshop on Deception, Fraud and Trust in Agent Societies. 2001.

[7] J.-J. CH. Meyer, R. J. Wieringa. Deontic logic: a Concise Overview. In: Deontic Logic in Computer Science: Normative System Specification. J.-J. CH. Meyer, R. J. Wieringa (eds.) 1993, Wiley.

[8] J. A. van Eck. A system of temporally relative modal and deontic predicate logic and its philosophical applications. Logique et Analyse, 100, 1982, 249-381.

[9] T. Maibaum, J. Fiadeiro, and S. Khosla. Temporal reasoning over deontic specifications. In Deontic logic in computer science: normative system specification, John Wiley and Sons Ltd., Chichester, 1994.

[10] A. N. Prior. Time and Modality. Oxford Press, 1957.

[11] M. Franceschet, A. Montanari, M. De Rijke. Model checking for combined logics with an application to mobile systems. Autom. Softw. Eng. 11(3), 289-321, 2004.

[12] A.J.I. Jones, M. Sergot. A Logical Framework. Open Agent Societies: Normative Specifications in Multi-Agent Systems, Wiley, 2003.

[13] A. Rotolo, G. Sartor, C. Smith. Good Faith in Contract Negotiation and Performance. IJBPIM Journal, Inderscience. 4(3) :154-173. doi: 10.1504/IJBPIM.2009.030983.

[14] A. Rotolo, C. Smith. Collective Trust and Normative Agents. Logic Journal of IGPL 2010; doi: 10.1093/jigpal/jzp076.

[15] S. K. Thomason. Semantic Analysis of Tense Logics. JSL, 35:150-158, 1972.

[16] R. Goldblatt. Mathematical Modal Logic: A View of its Evolution. Handbook of the History of logic. V. 6. 2005, Elsevier.

[17] Y. Venema. Temporal Logic. L. Goble ed., The Blackwell Guide to Philosophical Logic. Blackwell Publishers, USA. 203-223, 2001.

[18] P. Blackburn, M de Rijke, Y. Venema. Modal Logic. Cambridge, 2001.

[19] D. Elgesem. The Modal Logic of Agency. Nordic Journal of Philosophical Logic, 1997.

[20] G. Governatori, A. Rotolo. On the axiomatisation of Elgesem's logic of agency and ability. Journal Philosophical Logic, 2005, 34: 403-431. 
[21] M. Finger, D. Gabbay. Adding a temporal dimension to a logic system. Journal of Logic, Language and Information, 2:203-233, 1992.

[22] H. Herrestad, C. Krogh. Deontic Logic relativised to Bearers and Counterparties. J. Bing and O. Torvund eds., Anniversary Antology in Computers and Law -TANO 1995, pp 453-522.

[23] D. Memmo, G. Sartor, G. Quadri. 'Trust, reliance, good faith and the law', in Trust Management, Springer. 2003.

[24] H. MacQueen. 'Good faith in the Scots law of contract: an undisclosed principle?'. In Good Faith in Contract and Property Law, Hart. 1999.

[25] R. Brownsword, N. Bird, G. Howells. Good faith in contract: concept and context, in Good Faith in Contract, Ashgate. 1998.

[26] M. Storme. Good faith in the contents of contracts in European Private Law. Elec. Journ. Comparative Law, 2003.

[27] A. Musy. The good faith principle in Contract Law and the precontractual duty to disclose, in Global Jurist Advances. 2001.

[28] S. Whittaker, R. Zimmermann. Good Faith in European Contract Law. Cambridge University Press, 2000.

[29] T. Bolander, P. Blackburn. Terminating Tableau Calculi for Hybrid Logics extending K. www.elsevier.n1/locate/entcs. 2007.

[30] C. Areces, C. Monz, H de Nivelle, M. de Rijke. The Guarded Fragment. Ins and Outs. In J. Gerbrandy, et al eds., JFAK. Essays Dedicated to Johan van Benthem on the Occasion of his 50th Birthday, Vossiuspers, AUP, Amsterdam, 1999. 




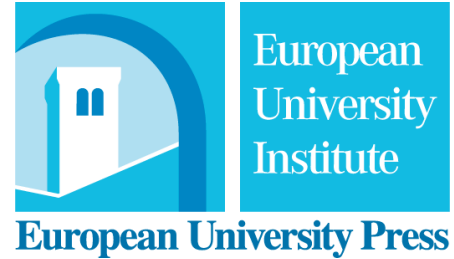

